\title{
Study of axion-like particles signals at Imaging Air Cherenkov Telescopes
}

\author{
Raquel Malta* and M. A. Leigui de Oliveira \\ Center for Natural Sciences and Humanities (CCNH) \\ Federal University of $A B C$ (UFABC) \\ 09210-580 Santo André, São Paulo, Brazil. \\ E-mail: raquel.malta@ufabc.edu.br
}

We propose a model for the flux of very-high energy (VHE) gamma rays at Earth in the energy region from $250 \mathrm{GeV}$ to $25 \mathrm{TeV}$. We take into account, along the path from the source to our Galaxy, the attenuation resulting from the photon interactions with the extragalactic background light (EBL), and the gamma-ray oscillations into axion-like particles (ALPs) due to their interactions with extragalactic magnetic field (EMF). We also discuss the expected increases in the gamma-ray flux to be observed by Imaging Air Cherenkov Telescopes (IACTs), if ALPs are confirmed by astrophysics as dark matter particles.

Key words: Axion-like particles, universal transparency of gamma rays, Imaging Air Cherenkov Telescopes.

36th International Cosmic Ray Conference -ICRC2019-

July 24th - August 1st, 2019

Madison, WI, U.S.A.

\footnotetext{
${ }^{*}$ Speaker.
} 


\section{Introduction}

\subsection{Gamma-ray astronomy}

Gamma-ray astronomy is the astronomical observation of gamma rays, the most energetic form of electromagnetic radiation, with photon energies above $100 \mathrm{keV}$. The mechanisms for gamma-ray emissions are diverse, mostly identical with those emitting X-rays but at higher energies, including electron-positron annihilation, the inverse Compton effect, and in some cases also the decay of radioactive material (gamma decay) in space reflecting extreme events such as supernovae and the behaviour of matter under extreme conditions, as in pulsars and blazars [1]. Electromagnetic radiation which falls upon Earth carries information about several aspects of universe. Especially the very energetic ones provide the understanding of physics of cosmic-ray acceleration in galactic and extragalactic objects, helping us to discover new systems far away from our galaxy and which phenomena does occur in these environments. VHE gamma rays point back directly to their sources and can directly reveal the production sites of energetic cosmic rays.

VHE photons $(100 \mathrm{GeV}-100 \mathrm{TeV})$ ejected from sources like supernovae remnants or active galactic nuclei (AGN), travel through intergalactic medium and undergo electron-positron creation, which leads to the attenuation of the primary beam [2]. This suppression depends both on the observed energy and the source distance, which can be measured by the source redshift. According to pair-production cross section, for $10 \mathrm{GeV}<E<10^{5} \mathrm{GeV}$, photons interact mainly with EBL, for $10^{5}<E<10^{10} \mathrm{GeV}$, cosmic microwave background (CMB) plays the leading role on the pair production process, and if $E>10^{10} \mathrm{GeV}$ the interaction with radio background (RB) becomes dominant. The photon survival probability can be measured as it follows [3]:

$$
P_{\gamma \gamma}\left(E_{0}, z_{s}\right)=e^{-\tau_{\gamma}\left(E_{0}, z_{s}\right)},
$$

where $\tau_{\gamma}$ is the optical depth that quantifies the dimming of the source at a redshift $z_{s}$ and $E_{0}$ is the observed energy. Evaluating the optical depth, equation 1.1 turns:

$$
P_{\gamma \gamma}(E, D)=e^{-D / \lambda_{\gamma}(E)}
$$

where $\mathrm{D}$ is the distance travelled by the photon and $\lambda_{\gamma}(E)$ is the photon mean free path.

The standard picture of pair-production absorption in the EBL seems to be incomplete since the expected opacity of the intergalactic space limits the mean free path of $\mathrm{TeV}$ gamma rays do not exceed 100 of megaparsecs, but $\mathrm{TeV}$ photons from numerous distant sources have been detected, causing an increasing slope of flux for $\tau \geq 1$. This implies that some novel astrophysical phenomena should affect long-distance propagation of gamma rays [4]. A scenario in which a part of energetic photons is converted to an inert particle along the way and reconverts back before reaching the observer. One of the explanations is a mixing between gamma rays and a light pseudoscalar fundamental boson, the so-called axion-like particle (ALP), which can, in principle, propagate largely unimpeded until reconverting in gamma-rays, inducing a hardening of the observed spectrum.

\subsection{Gamma-ray shower}

When an energetic cosmic ray falls upon Earth's atmosphere it triggers a chain-reaction particle cascade known as extensive air shower (EAS). An EAS is formed due to successive collisions 
between nucleons and the decays of its products. From the collisions of the primary cosmic ray with nucleons in the upper atmosphere, pions are produced. Neutral pions decay into two photons which interact with nuclear fields producing electron-positron pairs. Electrons and positrons in turn may produce more gamma-rays by bremsstrahlung - this is the electromagnetic component of the EAS. Charged pions decay into a muon and a muonic neutrino and this starts the muonic component, the most penetrating and most present at the ground level. A small portion of protons, neutrons, pions, and kaons are produced in the central axis of the shower, forming the hadronic component. Gamma-ray showers are mainly formed by the electromagnetic component. When charged particle travels through the air with a speed greater than the phase velocity of light for this medium, there is electromagnetic radiation emission, the Cherenkov radiation, which is the electromagnetic equivalent of a sonic boom. Cherenkov light may be produced in the air shower, with the maximum emission occurring when the number of particles in the cascade is the largest, at an altitude of $\approx 10$ $\mathrm{km}$ for primary energies of $100 \mathrm{GeV}-1 \mathrm{TeV}$, for example. Following absorption and scattering in the atmosphere, the Cherenkov light at ground level peaks at a wavelength $\lambda \approx 300-350 \mathrm{~nm}$, in the ultraviolet (UV) region of the electromagnetic spectrum. The photon density is typically $\approx 100$ photons $/ \mathrm{m}^{2}$ at $1 \mathrm{TeV}$, arriving in a brief flash lasting between 5 and $20 \mathrm{~ns}$. This Cherenkov pulse can be detected using large reflecting surfaces to focus light on to sensitive photon detectors, called imaging air-Cherenkov telescopes (IACTs).

\section{Imaging Air Telescopes}

The IACT works by imaging the very short flash of Cherenkov radiation produced when a VHE gamma ray strikes the atmosphere. The Cherenkov light is beamed around the direction of the incident primary particle and illuminates on the ground an area of about $250 \mathrm{~m}$ diameter, often referred to as the Cherenkov light pool. The image obtained shows the track of the air shower, which points back to the celestial object where the incident gamma ray came. With a single telescope providing a single view of a shower, it is difficult to predict its real structure, so in order to reconstruct the exact geometry of the shower multiple telescopes are used.

\subsection{H.E.S.S.}

H.E.S.S. (High Energy Stereoscopic System) is a system of IACTs located in Namibia in 1800 $\mathrm{m}$ above sea level, in an area well known for its excellent optical quality. The current Phase II H.E.S.S.has five telescopes: four from Phase I and one larger telescope constructed in the center of the array. Each telescope of phase I has a $12 \mathrm{~m}$ mirror and they are arranged in form of a square with $120 \mathrm{~m}$ side length. The larger telescope has a $28 \mathrm{~m}$ mirror, which added $600 \mathrm{~m}^{2}$ to the detection area. In its concept, the H.E.S.S. II camera contains 2048 PhotoMultiplier Tubes (PMTs) grouped into 16-PMT drawers which also contain the electronics for signal storage, signal digitization, triggering and readout. The lower threshold for gamma-ray detection in the H.E.S.S. system is around $10 \mathrm{GeV}$ and the upper threshold $10 \mathrm{TeV}$.

\subsection{MAGIC}

MAGIC (Major Atmospheric Gamma-ray Imaging Cherenkov telescopes) is a system of two IACTs situated at the Roque de los Muchachos Observatory (La Palma, Canary Islands) at about 


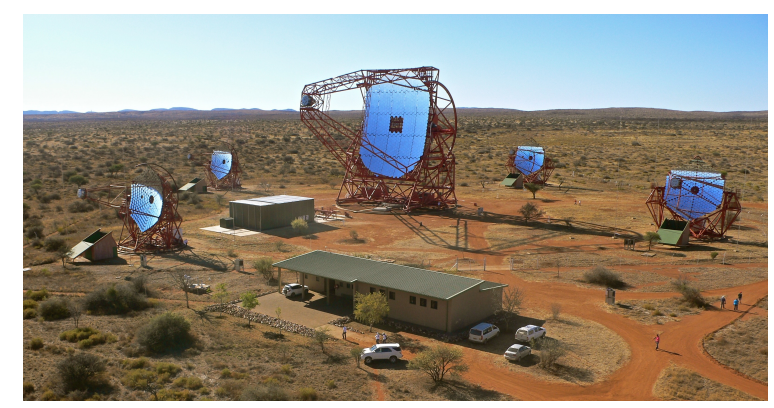

Figure 1: Overview of H.E.S.S. II telescopes array. [5]

$2200 \mathrm{~m}$ above sea level. The first telescope started routine operation in 2004 and the second one, built at a distance of $85 \mathrm{~m}$ from the first one, started taking data in 2010. Each telescope has an

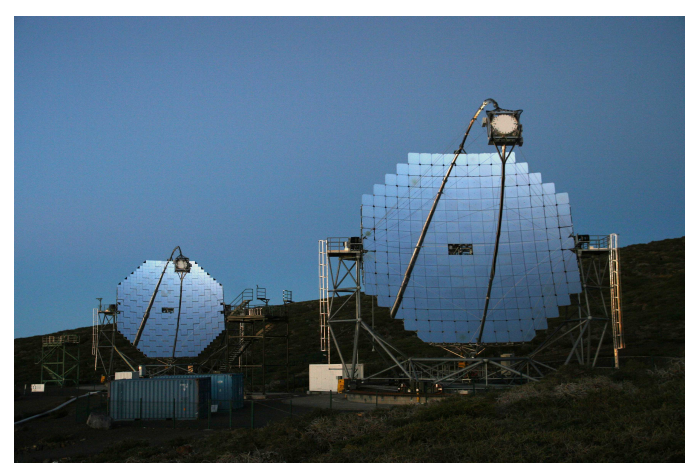

Figure 2: The two MAGIC telescopes at the Roque de los Muchachos Observatory. [6]

active reflective area of $236 \mathrm{~m}^{2}$ made of square mirrors. The cameras of both MAGIC telescopes consist of 1039 PMTs, arranged in a circle of about 1 meter diameter, which gives each camera a field of view in the sky of 3.5 degrees. All PMTs have an effective quantum efficiency of 25 to $35 \%$, depending on the wavelength. The lower threshold for gamma-ray detection in MAGIC is around $30 \mathrm{GeV}$ and the upper threshold is $100 \mathrm{TeV}$. The MAGIC telescopes are currently run by an international collaboration of about 165 astrophysicists from 24 institutions and consortia from 11 countries.

\subsection{VERITAS}

VERITAS (Very Energetic Radiation Imaging Telescope Array System) is an array of four telescopes located at Fred Lawrence Whipple Observatory, at 1268 meters above sea level. The first light with the complete array was in 2007. VERITAS telescopes have a $12 \mathrm{~m}$ optical reflector with an approximate separation of $100 \mathrm{~m}$ from each adjacent telescope.

Each of the individual telescopes have a 39 feet aperture, 350 mirrors on each dish, and a 3.5 degree field of view. VERITAS, like the other IACTs, are most sensitive to the highest energy cosmic rays, its full range of sensitivity is from $85 \mathrm{GeV}$ to over $30 \mathrm{TeV}$. 


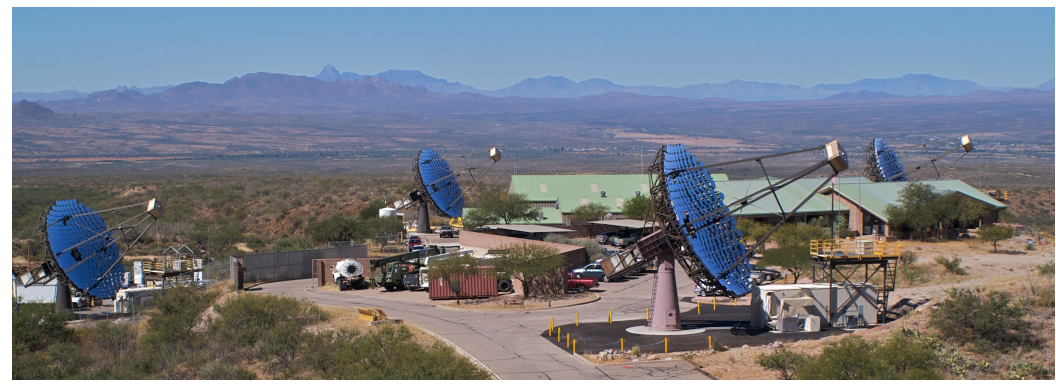

Figure 3: VERITAS array of four telescopes. [7]

\subsection{Cherenkov Telescope Array (CTA)}

Cherenkov Telescope Array will be the next generation ground-based gamma-ray telescopes for very high energy $(\mathrm{GeV}-\mathrm{TeV})$ astronomy, currently in its pre-construction phase and forseen to be operative a few years from now. The array comprise different numbers of telescopes of three different sizes: the Large-Size Telescope (LST), which will cover the unique low energy sensitivity of CTA between 20 and $150 \mathrm{GeV}$, the Medium-Size Telescope (MST), with sensitivity in the core energy range of CTA - from about $150 \mathrm{GeV}$ to $10 \mathrm{TeV}$ and Small-Size Telescope (SST), which is sensitive to the highest energy gamma rays, between a few $\mathrm{TeV}$ and $300 \mathrm{TeV}$. (Fig. 2.4).

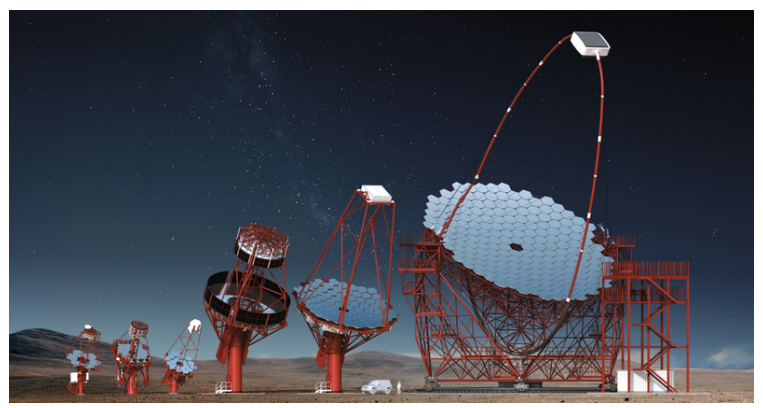

Figure 4: A comparative illustration of the three telescopes sizes [8]

The CTA Observatory should consist in two sites, one in the southern hemisphere, more precisely in the Atacama Desert at 2365 meters above sea level, and one in northern hemisphere, at Roque de los Muchachos Observatory, Canary Islands. The southern site will cover the center of the galactic plane and see many nearby galactic sources, some of which will produce $\mathrm{PeV}$ gamma rays. While the southern hemisphere array will span the entire energy range of CTA, the northern hemisphere array will be more limited in size and will focus on the low- and mid-energy ranges, for this reason the northern hemisphere site will not host any SSTs.

The aim of CTA is to make progress in over existing experiments in several science areas like SuperNova Remnants (SNRs) and cosmic ray, star-forming history of the universe, black holes, dark matter and more [8] through significant improvements in sensitivity and angular and temporal resolution, covering a wider energy range. CTA will be about a factor of 10 more sensitive than any existing instrument. As a consequence it will, for the first time, allow detection and in-depth study of large samples of known source types, it will explore a wide range of classes of suspected 
gamma-ray emitters and be sensitive to possible new phenomena that lie beyond the sensitivity of current instruments.

\section{Axions and Axion-like particles}

The axion a very light pseudo-Nambu-Goldstone boson of the broken $U(1)_{P Q}$ symmetry, proposed by Roberto Peccei and Helen Quinn [9] to solve the strong CP problem. Spontaneous breaking symmetry should leave a Nambu-Goldstone boson, but since this is an anomalous symmetry, this boson is not massless. Axions would have no electric charge, a very small mass and very low interaction cross-sections for strong and weak forces. An important aspect of axions (represented by the field $a$ ) is that they can couple to photons (represented by $\vec{E}$ ) in the presence of an external magnetic field $\vec{B}$. There are strong theoretical, astrophysical and cosmological motivations for models postulating the existence of additional particles which behave just as axions, the so-called Axion-Like Particles (ALPs) [10]. ALPs arise from spontaneous breaking of $U(1)_{X}$ symmetries, and their production is related to Primakoff effect inside the nucleus of astrophysical objects, just like the Sun. In this paper, we will consider this more general group. As a result of the photon-ALP coupling, a photon can oscillate into an ALP and vice-versa in an external magnetic field [11]. The oscillation probability is described by the equation 3.1 [12]:

$$
P_{\gamma a} \simeq \sin ^{2}\left(\frac{g_{a \gamma} B}{2} z\right)
$$

where $g_{a \gamma}$ is the coupling constant and $z$ is called domain, and it is equivalent to the total distance traveled by the particle. While for axions there exists a relation between the coupling constant and the axion mass $m_{a}$, in general $g_{a \gamma}$ and $m_{a}$ are unrelated for ALPs.

ALP-photon propagation over cosmological distance is strongly affected by the magnetic field structure, being a possible explanation for the anomalous transparency problem since radiation from AGNs travels in the form of ALPs for a significant fraction of distance (without producing pairs) and converts back to photons before their detections. The lower threshold of coupling constant $g_{a \gamma}$ to search effects of this anomalous transparency is $10^{-12}$ [13].

\section{Methodology}

We developed a computer simulation written in $\mathrm{C}++$ language which describes the processes that occur with photons along its path from Centaurus A to our galaxy regarding pair production process and photon x ALP mixing. First of all we used a Monte Carlo algorithm to simulate a power law spectrum of AGN, considering 1 million photons with energy ranging from $250 \mathrm{GeV}$ to $25 \mathrm{TeV}$, and three different slopes $-2.00,2.35,2.73$. These files were read by the code, which is divided according to the region of propagation: source galaxy, intergalactic medium and Milky Way, since the regions have different magnetic fields. Figure 5 shows a schematic representation of the problem and some possible events.

For each region, we calculated the number of electron-positron production and photon-ALP conversions. It is important to emphasize that the output data of one region is the input of the subsequent region. At the end of each step, we produced the spectra of each type of particle to observe the 


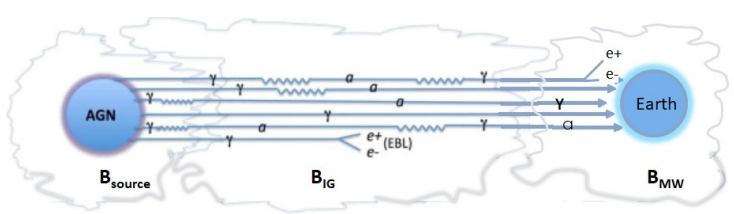

Figure 5: Some photon/ALP conversions that can occur since the emission of photons from a cosmological source until its arrival on Earth. Adapted from [14]

conversions that occurred in that specific region. The final spectrum contains the primary photons in the top of the atmosphere which will trigger gamma-ray air showers and produce Cherenkov radiation.

\section{Results}

Here we present the particles output flux in each region of propagation as a result of the simulated phenomena. First, the primary spectrum can be observed in figure 6 .

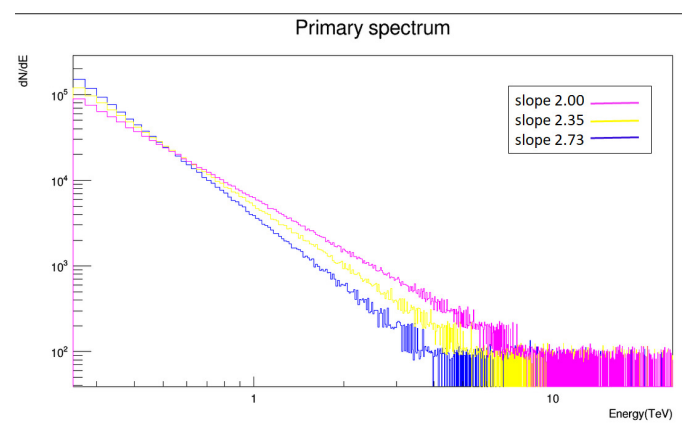

Figure 6: Primary spectrum with 1 million photons simulated with three different slopes.

\subsection{AGN}

Since for the three slopes the results are similar, we choose only one to illustrate the general scenario. We can infer that the coupling constant makes all the difference in photon x ALP
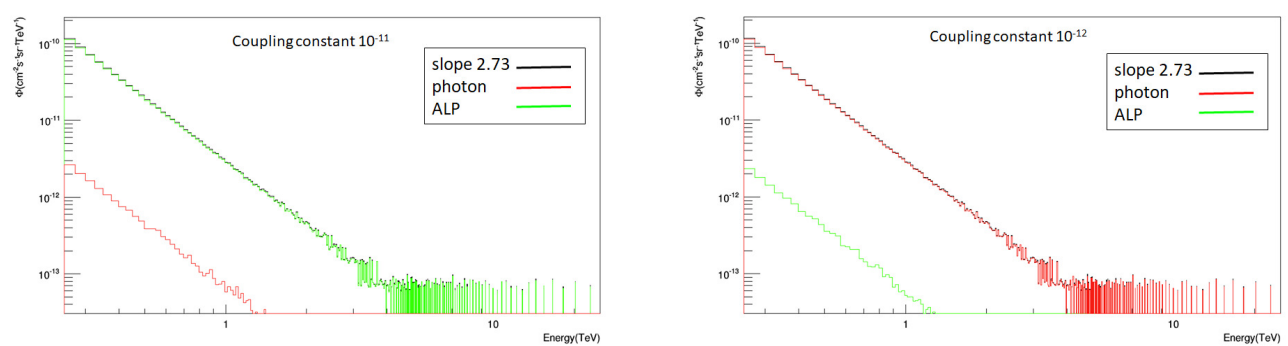

Figure 7: AGN output for a 2.73 slope and coupling constant $g_{a \gamma}=10^{-11}$ (left) and $g_{a \gamma}=10^{-12}$ (right) with non-attenuated photons and ALPs created along the path. 
oscillation. Since the oscillation probability is described by a sine-squared function, using AGN parameters $\left(B=10^{-5} \mathrm{G}\right.$ and $\left.z=2.83 \cdot 10^{20} \mathrm{~m}\right)$ for $g_{a \gamma}=10^{-11}$, we are close to a maximum, and so almost all of the photons are converted into ALPs. For $g_{a \gamma}=10^{-12}$ we have a low conversion and the output number of photons is almost equal to the primary spectrum.

\subsection{Intergalactic Medium}

Since the intergalactic magnetic field is a factor $10^{4}$ lower then the AGN magnetic field, the oscillation is severely suppressed and there is almost no conversion or reconversion. In this region we can notice pair production process due to interaction with EBL and so the total number of photons is decreased.
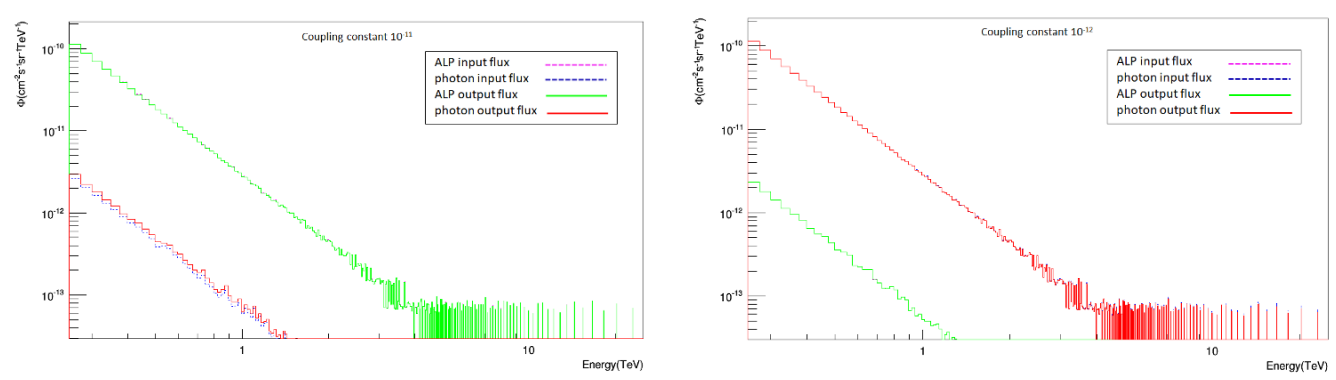

Figure 8: Intergalactic output for a 2.73 slope and coupling constant $g_{a \gamma}=10^{-11}$ (left) and $g_{a \gamma}=10^{-12}$ (right) showing the amount of photons and ALPs in comparison of photons arriving from AGN.

\subsection{Milky Way}

In this last region the magnetic field is $10^{4}$ times higher than in intergalactic medium so for $g_{a \gamma}=10^{-11}$ we can see a great conversion of ALP into photons, which increases the total photon flux in comparison with the input flux. The flux arriving on Earth is 13,9\% photons and $86 \%$ ALP (in comparison with the primary flux). For $g_{a \gamma}=10^{-12}$ the composition is $97,8 \%$ photons and $2,12 \%$ with no significant changes in relation to the previous region.
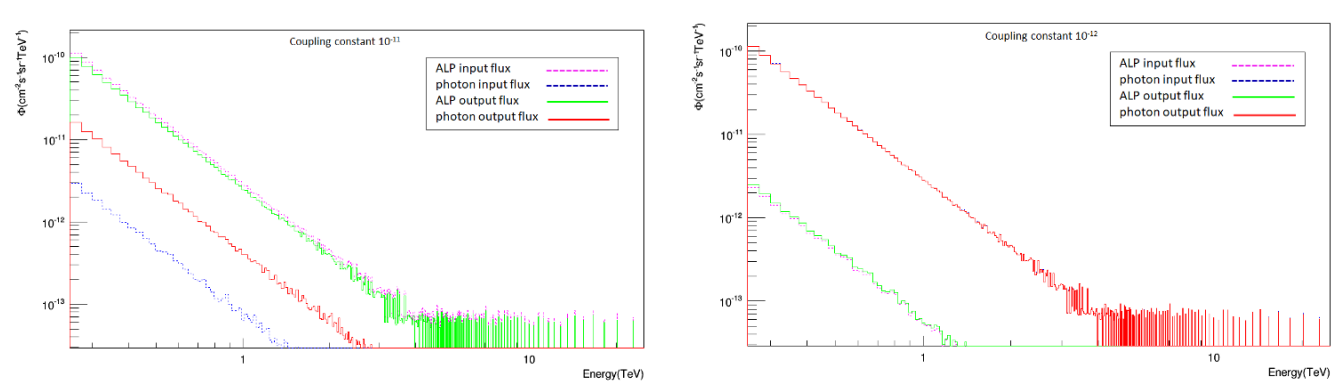

Figure 9: Flux arriving in Earth for a 2.73 slope and coupling constant $g_{a \gamma}=10^{-11}$ (left) and $g_{a \gamma}=10^{-12}$ (right) with photons that could be detected and ALPs 


\section{Conclusion}

Through this study we could see that for photons with energy from $250 \mathrm{GeV}$ to $25 \mathrm{TeV}$, ejected from Centaurus A, pair production is not a dominant process and occurs in a low scale. For coupling constant $g_{a \gamma}=10^{-12}$ oscillation process is severely suppressed, and the number of photons arriving on Earth is almost the number produced in AGN. For coupling constant $g_{a \gamma}=10^{-11}$ oscillation is a dominant effect and we have a boost in photon flux due to a high reconversion in Milky Way. We would like to acknowledge the São Paulo Research Foundation (FAPESP), grant n 2015/15897-1, for the support.

\section{References}

[1] Paredes, Josep M.; Reimer, Olaf; Torres, Diego F, “The Multi-Messenger Approach to High-Energy Gamma-Ray Sources: Third Workshop on the Nature of Unidentified High-Energy Sources," Springer. p. 180. ISBN 978-1-4020-6118-9.

[2] D. Horns and M. Meyer, "Indications for a pair-production anomaly from the propagation of VHE gamma-rays," JCAP 1202, 033 (2012).

[3] A. De Angelis, G. Galanti and M. Roncadelli, "Transparency of the Universe to gamma rays," Mon. Not. Roy. Astron. Soc. 432 (2013) 3245.

[4] G. I. Rubtsov and S. V. Troitsky, "Breaks in gamma-ray spectra of distant blazars and transparency of the Universe,” JETP Lett. 100, no. 6, 355 (2014) Pisma Zh. Eksp. Teor.Fiz. 100, no. 6, 397 (2014).

[5] Available from: https://commons.wikimedia.org/wiki/File:HESS_II_gamma_ray_experiment_five_telescope_array.jpg

[6] Highlights of the MAGIC telescopes - Scientific Figure on ResearchGate. Available from: https://www.researchgate.net/figure/A-picture-of-the-two-MAGIC-telescopes-at-the-Roque-de-losMuchachos-observatory_fig4_228467537

[7] Available from: https://commons.wikimedia.org/wiki/File:VERITAS_array.jpg/media/File:VERITAS_array.jpg

[8] B. S. Acharya et al. [Cherenkov Telescope Array Consortium], "Science with the Cherenkov Telescope Array," arXiv:1709.07997 [astro-ph.IM].

[9] R. D. Peccei and H. R. Quinn, “CP Conservation in the Presence of Pseudoparticles," Phys. Rev. Lett. 38 (1977) 1440.

[10] K.A. Olive et al. (Particle Data Group), “Axions and other similar particles,” Chin. Phys. C38, 090001 (2014)

[11] C. Wang and D. Lai, “Axion-photon Propagation in Magnetized Universe,” JCAP 1606, no. 06,006 (2016).

[12] C. D. R. Carvajal, A. G. Dias, C. C. Nishi and , B. L. Sánchez-Vega, "Axion like particles and the inverse seesaw mechanism," JHEP 2015, 69 (2015).

[13] A. G. Dias, A. C. B. Machado, C. C. Nishi, A. Ringwald and P. Vaudrevange, "The Quest for an Intermediate-Scale Accidental Axion and Further ALPs," JHEP 1406, 037 (2014

[14] M. Doro et al. [CTA Consortium], "Dark Matter and Fundamental Physics with the Cherenkov Telescope Array," Astropart. Phys. 43 (2013) 189. 